\title{
Sport participation and related head injuries following craniosynostosis correction: a survey study
}

\author{
Aaron M. Yengo-Kahn, MD,1,2 Oluwatoyin Akinnusotu, BS, ${ }^{2,3}$ Alyssa L. Wiseman, MA, MS, ${ }^{2}$ \\ Muhammad Owais Abdul Ghani, MBBS, ${ }^{2,4}$ Chevis N. Shannon, DrPH, MBA, MPH, ${ }^{1,2}$ \\ Michael S. Golinko, MD, ${ }^{4}$ and Christopher M. Bonfield, MD ${ }^{1,2}$
}

\begin{abstract}
Departments of ${ }^{1}$ Neurological Surgery and ${ }^{4}$ Plastic Surgery, Vanderbilt University Medical Center; ${ }^{2}$ Surgical Outcomes Center for Kids, Monroe Carell Jr. Children's Hospital at Vanderbilt University; and '3Meharry Medical College School of Medicine, Nashville, Tennessee
\end{abstract}

\begin{abstract}
OBJECTIVE Craniosynostosis (CS) affects about 1 in 2500 infants and is predominantly treated by surgical intervention in infancy. Later in childhood, many of these children wish to participate in sports. However, the safety of participation is largely anecdotal and based on surgeon experience. The objective of this survey study was to describe sport participation and sport-related head injury in CS patients.
\end{abstract}

METHODS A 16-question survey related to child/parent demographics, CS surgery history, sport history, and sportinduced head injury history was made available to patients/parents in the United States through a series of synostosis organization listservs, as well as synostosis-focused Facebook groups, between October 2019 and June 2020. Sports were categorized based on the American Academy of Pediatrics groupings. Pearson's chi-square test, Fisher's exact test, and the independent-samples t-test were used in the analysis.

RESULTS Overall, 187 CS patients were described as $63 \%$ male, $89 \%$ White, and $88 \%$ non-Hispanic, and $89 \%$ underwent surgery at 1 year or younger. The majority (74\%) had participated in sports starting at an average age of 5 years (SD 2.2). Of those participating in sports, contact/collision sport participation was most common (77\%), and $71 \%$ participated in multiple sports. Those that played sports were less frequently Hispanic $(2.2 \%$ vs $22.9 \%, p<0.001)$ and more frequently had undergone a second surgery (44\% vs $25 \%, p=0.021)$. Only 9 of $139(6.5 \%)$ sport-participating CS patients suffered head injuries; 6 (67\%) were concussions and the remaining 3 were nondescript but did not mention any surgical needs.

CONCLUSIONS In this nationwide survey of postsurgical CS patients and parents, sport participation was exceedingly common, with contact sports being the most common sport category. Few head injuries (mostly concussions) were reported as related to sport participation. Although this is a selective sample of CS patients, the initial data suggest that sport participation, even in contact sports, and typically beginning a few years after CS correction, is safe and commonplace.

https://thejns.org/doi/abs/10.3171/2021.1.FOCUS20970

KEYWORDS craniosynostosis; craniofacial reconstruction; sport participation; sport-related head injury

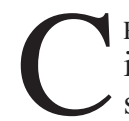
RANIOSYNOSTOSIS (CS) is a craniofacial abnormality resulting from premature fusion of the cranial sutures. The overall incidence of CS has been estimated at 1 in 2500 births. ${ }^{1-3}$ Isolated single-suture CS is most common, representing $>80 \%$ of cases, with multisuture and syndromic CS each contributing less than $10 \%$ of cases. ${ }^{1-3}$ Both for cosmesis and to avoid complications associated with the possible development of intracranial hypertension, children with CS typically undergo surgical correction in infancy. ${ }^{4-7}$ The optimal timing of intervention is generally within the 1st year of life in order to mitigate developmental delay and orbital/calvarial deformities?

While a small subset of patients may demonstrate subtle deficits on neuropsychological assessments, most children who undergo single-suture CS correction in early infancy demonstrate normal neurological development. ${ }^{8}$ Given the frequently normal postsurgical neurodevelopment, many of these children (and parents) wish to participate in sports with their peers.

There is limited evidence regarding the safety of sports

ABBREVIATIONS CS = craniosynostosis; REDCap = Research Electronic Data Capture; TBI = traumatic brain injury; UK = United Kingdom.

SUBMITTED November 4, 2020. ACCEPTED January 14, 2021.

INCLUDE WHEN CITING DOI: 10.3171/2021.1.FOCUS20970. 
in adolescents following CS surgery. Given the extent of the surgery required and high incidence of sport and recreation-related head injury, ${ }^{9}$ both providers and parents may be anxious regarding the prospect of enrolling a child with previously corrected CS in sports, or they may limit the range of sports in which the child is allowed to participate. These concerns, while reasonable, lack any foundational evidence. In fact, sparse evidence on the topic suggests that, in general, CS patients experience a similar rate of traumatic brain injury (TBI) to non-CS children. ${ }^{10}$ Recently a small study of postsurgical CS patients in the United Kingdom (UK) found that many CS patients surveyed had participated in sports with only a few minor head injuries reported. ${ }^{11}$

Despite these initial investigations, physicians and parents continue to rely on anecdotal recommendations. The objective of the current survey study was to broadly describe the sport participation experience of children who have undergone surgical correction of CS, including whether participation led to any significant head injuries. We hypothesized that a wide spectrum of sport participation would be reported with few catastrophic head injuries.

\section{Methods \\ Study Design}

A survey-based cross-sectional study was designed to estimate the frequency of sport participation and any related head injuries for children following CS repair. The study received approval from the Vanderbilt University Medical Center institutional review board. Electronic consent was required from participants prior to accessing the survey questions. The survey was open from October 1, 2019, to June 30, 2020.

\section{Participants}

Any person who had previously undergone surgical correction of CS and those who were parents of such children were eligible to participate. Those who did not complete the survey were excluded. The survey was distributed via an email listserv maintained by FACES: The National Craniofacial Association. ${ }^{12}$ The survey was also deposited on the "walls" (i.e., message boards) of several Facebook (www.facebook.com) communities, including Cranio Kids-Craniosynostosis Support, Adult Craniosynostosis Survivors, Vandy Cranio Moms, Craniosynostosis Support for Parents and Guardians, Craniosynostosis, and Craniosynostosis Endoscopic Surgery.

\section{Survey Design}

The survey was built using the Research Electronic Data Capture (REDCap) web application. ${ }^{13}$ Study data were collected and managed using REDCap electronic data capture tools hosted at Vanderbilt University Medical Center. ${ }^{13}$ REDCap is a secure, web-based software platform designed to support data capture for research studies, providing 1) an intuitive interface for validated data capture; 2) audit trails for tracking data manipulation and export procedures; 3) automated export procedures for seamless data downloads to common statistical packages; and 4) procedures for data integration and interoperability with external sources. At the time of the survey design, no prior empirical studies on the subject of sport participation following CS repair existed. Therefore, survey elements were designed to maximize participation by minimizing survey completion time. We tested the survey for clarity and time of completion prior to distribution. The survey contained the study team's contact information to allow feedback on the clarity of the survey or for questions. While a plan was made to pause enrollment if survey refinement was needed, no questions or concerns arose and no survey revisions were performed during the study. The full survey is available in the Supplemental Material.

Survey elements identified the respondent as either the patient or parent and confirmed that the respondent or their child had undergone surgical correction for CS in the past. All respondents were asked for patient demographic information (current age, sex, race, and ethnicity). Child age at their first surgery was reported in years and recorded as 0 years if surgery was performed prior to 1 year of age. Respondents also reported whether or not additional surgeries were needed for CS and the age at which the second surgery occurred (when applicable).

The primary outcome of interest was whether or not the child participated in sports after the initial surgery, which was simply recorded as yes or no. Those responding "yes" were then prompted to describe sport participation and ages at the time of participation as a free-text response. Those who reported sport participation were also asked if they "experienced a major head injury while playing sports." If they responded "yes," they were prompted to provide a free-text description of the head injury.

\section{Statistical Analysis}

To facilitate analysis, free-text responses were individually reviewed and coded. The age at first surgery was categorized as $<1$ year or $\geq 1$ year. Sport responses were provided as free text and subsequently grouped according to the American Academy of Pediatrics categories of sport: noncontact, low contact, contact/collision, and extreme (e.g., BMX, skateboarding, snowboarding, and skiing). ${ }^{14}$ If the respondent reported multiple sports, the recorded category reflected the highest contact level of participation. Additionally, each sport was extracted for completeness, as many respondents reported participation in several sports. The age (years) at sport participation was defined as the earliest age of participation in any sport. Information on type of head injury was converted from free-text responses to either concussion or other head injury, based on whether or not the word "concussion" was noted in the response.

Survey responses were stratified by sport participation status, and those who reported participation were substratified by head injury status. As a substantial proportion of patients reported second surgeries, a supplemental analysis was performed comparing sport participation reported by those who did and those did not have second surgeries. Sample size was dictated by available responses. No data were imputed. Categorical variables are presented as frequencies and proportion, and continuous variables are presented as means with standard deviations. The thresh- 
TABLE 1. Craniosynostosis patients stratified by sport participation after surgical correction

\begin{tabular}{lcccc}
\hline & Play Sports $(\mathrm{n}=139)$ & Did/Do Not Play $(\mathrm{n}=48)$ & $\mathrm{p}$ Value* & Overall $(\mathrm{n}=187)$ \\
\hline Male sex & $88(63.3)$ & $29(60.4)$ & 0.72 & $117(62.6)$ \\
\hline White race & $126(90.6)$ & $41(85.4)$ & 0.31 & $167(89.3)$ \\
\hline Ethnicity & & & $<0.001$ & \\
\hline Hispanic & $3(2.2)$ & $11(22.9)$ & & $14(7.5)$ \\
\hline Non-Hispanic & $131(94.2)$ & $34(70.8)$ & & $165(88.2)$ \\
\hline Unknown/other & $5(3.6)$ & $3(6.3)$ & & $8(4.3)$ \\
\hline Age $\leq 1$ yr at op & $126(90.6)$ & $41(85.4)$ & 0.31 & $167(89.3)$ \\
\hline 2nd op & $61(43.9)$ & $12(25.0)$ & 0.021 & $73(39.0)$ \\
\hline Mean age at 2nd op \pm SD, yrs & $4.90 \pm 4.1$ & $3.58 \pm 3.18$ & $0.23 \dagger$ & $4.68 \pm 3.9$ \\
\hline $\begin{array}{l}\text { Values represent the number of patients (\%) unless stated otherwise. } \\
\text { * Pearson's chi-square test unless otherwise noted. }\end{array}$ & & & \\
$\dagger$ & & &
\end{tabular}

old for statistical significance was set a priori as $\mathrm{p}<0.05$. Pearson's chi-square test and independent-sample t-tests were used to assess categorical and continuous variables, respectively. All statistical analyses were performed in SPSS Statistics (version 26, IBM Corp.).

\section{Results}

The survey link was opened by 221 respondents; 32 were excluded, as they or their child had not undergone surgery. Of the 189 eligible respondents, 187 (98.9\%) completed the full set of questions. Of the respondents, $89.8 \%$ were parents of children who underwent CS surgery, and $10 \%$ were patients themselves. At the time of the survey, the children who had undergone surgery (herein referred to as "patients") were a mean age of 13.2 years (SD 9.65 years, median 11 years) with a range of 53 years (2-55 years). Overall, patients were mostly White $(89.3 \%)$, nonHispanic (88.2\%), and male (62.6\%). Approximately $90 \%$ were younger than 1 year at the time of their first surgery. In total, 39\% underwent a second surgery at a mean age of 4.4 years.

\section{Sport Participation}

Overall, 139 of $187(74.3 \%)$ patients participated in sports following CS correction. The demographics and first surgery timing were generally similar for those who played sports compared with those who did not (Table 1). However, the distribution of ethnicity was dependent on sport participation status $(\mathrm{p}<0.001)$, with Hispanic patients overrepresented in the nonparticipation group. Those who played sports had more frequently undergone a second surgery $(43.9 \%$ vs $25 \%, \mathrm{p}=0.021)$.

Those patients who played sports started participating at a mean age of 5.1 years (SD 2.2 years), and most $(76.8 \%)$ participated in at least one contact/collision sport. The top 3 sports by frequency of participation were soccer (47.8\%), baseball/softball (38.8\%), and basketball (32.4\%). Table 2 provides a complete accounting of sports reported. CS patients who had undergone two or more surgeries reported similar participation in the most popular 5 sports, as well as in each sport category (Table 3).

\section{Head Injuries}

Of 139 patients who participated in sports, 9 (6.5\%) reportedly experienced a major head injury during sports. Of these injuries, $6(66.7 \%)$ were called "concussions" in free-text responses. There were no statistical differences in age, high contact level of sport participation, or multiple sport participation between those who had suffered head injuries and those who had not (Table 4). Three responses, reproduced in Table 5, detailed the "other" head injuries. Of those patients that reported second surgeries, $6(9.8 \%)$ reported head injuries, compared with $3(3.8 \%)$ of those patients reporting a single surgery $(\mathrm{p}=0.154)$.

\section{Discussion}

Using a cross-sectional survey study, sport participation appears very common among CS patients following surgical correction and is rarely complicated by a significant head injury. CS patients participated in a wide range of sports across all contact categories, with soccer being the most common. Concussions represented $66 \%$ of head injuries, and other injuries usually were also mild or vaguely described. Sport participation and especially team sport participation can be highly beneficial for children, ${ }^{15}$ and based on this cross-sectional study, the risks of head injury for CS patients do not appear to outweigh these benefits.

\section{Sport Participation}

To date, sport participation following CS surgery has been poorly described in the literature, and surgeons rely on anecdotal experience in order to provide counseling to patients. ${ }^{11}$ A small survey $(n=59)$ of postsurgical CS patients in the UK found a similarly high rate of sport participation (88\%), with the majority involved in noncontact or light-contact sports. ${ }^{11}$ Unfortunately, the categorization of contact levels is not completely described, and such a comparison across studies is difficult. However, soccer was included as a "light-contact" sport, and the reported $38 \%$ participation rate is generally similar to the participation rate we found for soccer alone. Similarly, participa- 


\section{TABLE 2. Sport participation}

\begin{tabular}{|c|c|}
\hline & No. of Patients (\%) \\
\hline \multicolumn{2}{|l|}{ Sport category* } \\
\hline Noncontact & $15(10.9)$ \\
\hline Low contact & $14(10.1)$ \\
\hline Collision/contact & $106(76.8)$ \\
\hline Extreme & $3(2.2)$ \\
\hline Participated in multiple sports & $99(71.2)$ \\
\hline \multicolumn{2}{|l|}{ Breakdown by sport $†$} \\
\hline Soccer & $66(47.8)$ \\
\hline Baseball/softball & $54(38.8)$ \\
\hline Basketball & $45(32.4)$ \\
\hline Football & $22(15.8)$ \\
\hline Dance/cheer & $16(11.5)$ \\
\hline Swim/dive & $15(10.8)$ \\
\hline Gymnastics & $12(8.6)$ \\
\hline Flag football (never full pads) & $10(7.2)$ \\
\hline Volleyball & $10(7.2)$ \\
\hline Martial arts & $9(6.5)$ \\
\hline Cross-country/track \& field & $6(4.3)$ \\
\hline Wrestling & $6(4.3)$ \\
\hline Other $\ddagger$ & $6(4.3)$ \\
\hline Golf & $5(3.6)$ \\
\hline Extreme§ & $3(2.2)$ \\
\hline Tennis & $3(2.2)$ \\
\hline Lacrosse & $1(0.7)$ \\
\hline
\end{tabular}

* Sport category is the highest level of contact reported. For one response, the sport was unknown, although the patient indicated involvement in multiple sports.

† Categories not mutually exclusive, and percentages will not sum to 100 . $\ddagger$ Includes archery $(n=1)$, "netball" $(n=1)$, and bowling $(n=1)$. $\S$ Includes skiing $(n=2)$, mountain biking $(n=2)$, parkour $(n=1)$, and skateboarding $(n=1)$. One child participates in 4 different extreme sports.

tion in "heavy-contact" sports (e.g., rugby) was reported at approximately $10 \%$, comparable to the $16 \%$ for American football participation found in the present study. Based on this recent study and our own results, sport participation appears generally common among CS patients.

Although Rotimi et al. previously found a correlation between increasing sport participation and decreasing contact potential, ${ }^{11}$ our results do not clearly replicate this trend. Instead, our results essentially mirror the top 5 sports for both youth and high school athletes in the US. ${ }^{16,17}$ While parental anxiety may have a role in which sports CS patients ultimately participate, ${ }^{11} \mathrm{CS}$ patients do seem to participate in sports in a pattern similar to their peers at a national level. Unfortunately, we were not able to interview parents or delve deeply into how these decisions are reached, and to what extent surgeon counseling plays a role. This remains an open avenue for further study, both for CS patients and in general for any child who has undergone a neurosurgical operation early in life.

Interestingly, we found that patients who participated in sports had more frequently undergone a second surgery.
TABLE 3. Craniosynostosis patients' sport participation stratified by multiple versus single surgery

\begin{tabular}{lccc}
\hline & $\geq 2$ Ops $(\mathrm{n}=61)^{*}$ & Single Op $(\mathrm{n}=77)$ & $\mathrm{p}$ Value $\dagger$ \\
\hline Sport category, $\mathrm{n}(\%)$ & & 0.094 \\
\hline Noncontact & $9(14.8)$ & $6(7.8)$ & \\
\hline Low contact & $7(11.5)$ & $7(9.1)$ & \\
\hline Contact/collision & $42(68.9)$ & $64(83.1)$ & \\
\hline Extreme & $3(4.9)$ & $0(0)$ & 0.204 \\
\hline Soccer & $25(41.7)$ & $41(52.6)$ & 0.214 \\
\hline Football & $7(11.5)$ & $15(19.2)$ & 0.195 \\
\hline Baseball/softball & $20(32.8)$ & $34(43.6)$ & 0.523 \\
\hline Basketball & $18(29.5)$ & $27(34.6)$ & 0.584 \\
\hline Dance/cheer & $6(9.8)$ & $10(12.8)$ & \\
\hline
\end{tabular}

${ }^{*}$ For one response, the sport was unknown, although the patient indicated involvement in multiple sports.

† Pearson's chi-square test.

Given the brief nature of the electronic survey, we are unable to describe the indications of these second surgeries. One possibility is that children who expressed interest in sport participation were more likely to undergo cranioplasty prior to sport participation, as the mean age at the second surgery is just slightly younger than the age at which they first participated in sports. To our knowledge, there are no consensus guidelines on which to base the decision to pursue cranioplasty. Previously, authors have suggested that persistent defects sized $2-2.5 \mathrm{~cm}^{2}$ may be considered "clinically relevant" and cranioplasty should be considered.6,18,19 Published rates of these relevant defects are generally around $20 \%-25 \%,{ }^{18,20}$ while we found that about $44 \%$ of CS patients who have participated in sports had a second surgery. While this may suggest a more aggressive approach toward providing cranioplasty for those children wishing to participate in sport, the high rate of second surgeries may be a result of survey selection biases where parents and patients with more complicated surgical courses are more likely to belong to support groups and online communities. These findings should provide further reassurance to families and surgeons, as even CS patients who undergo multiple surgeries report sport participation without an increased injury rate.

\section{Sport-Related Head Injuries}

We found that $6.5 \%$ of CS patients who participated in sports had sustained a sport-related head injury. None of these were clearly described as requiring surgery. One respondent mentioned a TBI resulting from a line drive in baseball, which can be a severe injury regardless of prior cranial operations. Similarly, the previous UK cohort demonstrated a $5.7 \%$ rate of sport-related head injury, none of which required surgery or hospitalization. ${ }^{11}$ While we do not have imaging data from these patients at the time of their head injuries, the descriptions of injuries suggest that only the one patient may have suffered a structural brain injury, let alone any form of TBI. The rarity of these events appears congruent with a prior study reporting an incidence of 0/100,000 for CS patients during a 2-year postop- 
TABLE 4. Sport-participating patient characteristics stratified by head injury status

\begin{tabular}{lccc}
\hline & $\begin{array}{c}\text { Head Injured } \\
(n=9)\end{array}$ & $\begin{array}{c}\text { Non-Head Injured } \\
(n=130)^{*}\end{array}$ & p Value† \\
\hline $\begin{array}{l}\text { Mean age enrolled in } \\
\text { sport } \pm \text { SD, yrs }\end{array}$ & $5.9 \pm 2.1$ & $5.0 \pm 2.2$ & $0.292 \ddagger$ \\
\hline $\begin{array}{l}\text { Sport category, } \mathrm{n}(\%) \\
\text { Noncontact }\end{array}$ & $0(0)$ & $15(9.5)$ & \\
\hline Low contact & $3(33.3)$ & $11(8.5)$ & \\
\hline Contact/collision & $6(66.7)$ & $100(77.5)$ & \\
\hline Extreme & $0(0)$ & $3(2.3)$ & \\
\hline $\begin{array}{l}\text { Participated in } \\
\text { multiple sports }\end{array}$ & $5(55.6)$ & $94(72.3)$ & 0.283 \\
\hline
\end{tabular}

* For one response, the sport was unknown, although the patient indicated involvement in multiple sports.

$†$ Pearson's chi-square test unless noted otherwise.

$\ddagger$ Student 2-sample t-test.

erative follow-up period. ${ }^{10}$ Summarily, CS patients appear to be at no increased risk of sport-related TBI.

\section{Limitations}

The limitations of this study are related to the brief survey-based design. First, selection bias in the form of response bias is introduced based on the mode of survey distribution, relying on Facebook community groups and organization listservs. Relying on these resulted in a rather homogeneous study group in terms of race and ethnicity. While CS is more common in White families in the US, the present study group is more similar to samples drawn from large tertiary care centers than a full US sample. ${ }^{2}$ The disproportionate representation of Hispanic patients we report in the group not participating in sports may be related to sampling rather than true differences in participation and requires further investigation.

Relying on survey data also may have resulted in only including patients and families who have had great or very poor experiences as they may be more likely to engage in online communities and survey opportunities. However, there were very few, if any, significant injuries reported, and if these sampling biases were prominent, more extreme scenarios would have been expected. Similarly, our cohort did report a high rate of second surgeries for CS that most likely reflects sampling biases inherent in recruiting respondents through online communities and support groups, which may contain a higher proportion of complex cases.

Finally, we purposefully used a brief survey to encourage completion, and while we succeeded in this goal with a $99 \%$ completion rate, we sacrificed some details that may have helped interpret the results. For example, we do not know what proportion of CS patients underwent surgery for single-suture synostosis versus syndromic or multisuture synostosis. However, our supplemental analysis suggests that those who underwent single surgeries (i.e., presumably nearly all single-suture CS) report similar sport participation patterns as those with two or more surgeries. Similarly, we do not have detailed information
TABLE 5. Free-text responses to "other head injuries"

"Traumatic brain injury while pitching-baseball_took a line drive to the skull."

"Gymnastics. Not sure what happened but her head swelled up and shifted."

"Head hit by ball being kicked."

on the nature of second surgeries to support the hypothesis that children are encouraged to undergo cranioplasty prior to participation. While the additional information is certainly of interest, these details would not dramatically change the primary study findings that CS patients can and do participate in a wide array of recreational activities without any suggestion of increased risk of TBI.

\section{Conclusions}

In this survey of postsurgical CS patients and parents, sport participation is exceedingly common, with soccer, baseball/softball, and basketball being the most frequently reported. Few head injuries (the majority being concussions) were reported as related to sport participation. Although from a selective sample of CS patients, the initial data suggest that sport participation, even in contact sports, and typically beginning a few years after CS correction, is safe and commonplace.

\section{Acknowledgments}

This study utilized REDCap, which is funded through NCATS/NIH grant support (UL1 TR000445), for survey and data management. There was no direct funding received or utilized for this study.

\section{References}

1. Boulet SL, Rasmussen SA, Honein MA. A population-based study of craniosynostosis in metropolitan Atlanta, 19892003. Am J Med Genet A. 2008;146A(8):984-991.

2. Sacks GN, Skolnick GB, Trachtenberg A, et al. The impact of ethnicity on craniosynostosis in the United States. J Craniofac Surg. 2019;30(8):2526-2529.

3. Johnson D, Wilkie AOM. Craniosynostosis. Eur J Hum Genet. 2011;19(4):369-376.

4. Stricker PA, Goobie SM, Cladis FP, et al. Perioperative outcomes and management in pediatric complex cranial vault reconstruction: a multicenter study from the Pediatric Craniofacial Collaborative Group. Anesthesiology. 2017;126(2):276-287.

5. Thompson DR, Zurakowski D, Haberkern CM, et al. Endoscopic versus open repair for craniosynostosis in infants using propensity score matching to compare outcomes: a multicenter study from the Pediatric Craniofacial Collaborative Group. Anesth Analg. 2018;126(3):968-975.

6. Anantheswar YN, Venkataramana NK. Pediatric craniofacial surgery for craniosynostosis: our experience and current concepts: Part -1. J Pediatr Neurosci. 2009;4(2):86-99.

7. Bruce WJ, Chang V, Joyce CJ, et al. Age at time of craniosynostosis repair predicts increased complication rate. Cleft Palate Craniofac J. 2018;55(5):649-654.

8. Chieffo D, Tamburrini G, Massimi L, et al. Longterm neuropsychological development in single-suture craniosynostosis treated early. J Neurosurg Pediatr. 2010; 5(3):232-237. 
9. Sarmiento K, Thomas KE, Daugherty J, et al. Emergency department visits for sports- and recreation-related traumatic brain injuries among children-United States, 2010-2016. MMWR Morb Mortal Wkly Rep. 2019;68(10):237-242.

10. Gilardino MS, Jandali S, Whitaker LA, Bartlett SP. Does the incidence of traumatic brain injury in children increase after craniofrontal surgery? J Craniofac Surg. 2011;22(4): $1284-1286$.

11. Rotimi O, Jung GP, Ong J, et al. Sporting activity after craniosynostosis surgery in children: a source of parental anxiety. Childs Nerv Syst. 2021;37(1):287-290.

12. FACES. The National Craniofacial Association. Accessed February 8, 2021. https://www.faces-cranio.org/

13. Harris PA, Taylor R, Thielke R, et al. Research electronic data capture (REDCap) - a metadata-driven methodology and workflow process for providing translational research informatics support. J Biomed Inform. 2009;42(2):377-381.

14. Rice SG. Medical conditions affecting sports participation. Pediatrics. 2008;121(4):841-848.

15. Logan K, Cuff S. Organized sports for children, preadolescents, and adolescents. Pediatrics. 2019;143(6):e20190997.

16. Project Play. State of Play 2019: Trends and Developments in Youth Sports. Aspen Institute. Accessed February 8, 2021. https://assets.aspeninstitute.org/content/ uploads/2019/10/2019_SOP_National_Final.pdf

17. National Federation of State High School Associations. NFHS. org. Accessed February 8, 2021. https://www.nfhs.org/sportsresource-content/high-school-participation-survey-archive/

18. Skolnick GB, Murthy S, Patel KB, et al. Long-term characterization of cranial defects after surgical correction for singlesuture craniosynostosis. Ann Plast Surg. 2019;82(6):679-685.

19. Posnick JC, Goldstein JA, Armstrong D, Rutka JT. Reconstruction of skull defects in children and adolescents by the use of fixed cranial bone grafts: long-term results. Neurosurgery. 1993;32(5):785-791.

20. Paige KT, Vega SJ, Kelly CP, et al. Age-dependent closure of bony defects after frontal orbital advancement. Plast Reconstr Surg. 2006;118(4):977-984.

\section{Disclosures}

The authors report no conflict of interest concerning the materials or methods used in this study or the findings specified in this paper.

\section{Author Contributions}

Conception and design: Yengo-Kahn, Wiseman, Shannon, Golinko, Bonfield. Acquisition of data: Akinnusotu, Wiseman, Owais Abdul Ghani. Analysis and interpretation of data: YengoKahn, Owais Abdul Ghani, Bonfield. Drafting the article: YengoKahn, Akinnusotu, Wiseman. Critically revising the article: Yengo-Kahn, Owais Abdul Ghani, Shannon, Golinko, Bonfield. Reviewed submitted version of manuscript: all authors. Approved the final version of the manuscript on behalf of all authors:

Yengo-Kahn. Statistical analysis: Yengo-Kahn. Administrative/ technical/material support: Wiseman, Shannon. Study supervision: Yengo-Kahn, Shannon, Golinko, Bonfield.

\section{Supplemental Information}

Online-Only Content

Supplemental material is available online.

Supplemental Material. https://thejns.org/doi/suppl/10.3171/ 2021.1.FOCUS20970.

\section{Previous Presentations}

This work was presented in abstract form at the Annual Meeting of the AANS/CNS Section on Pediatric Neurosurgery, December 2-4, 2020 (virtual).

\section{Correspondence}

Aaron M. Yengo-Kahn: Vanderbilt University Medical Center, Nashville,TN. a.yengo@vumc.org. 